A major purpose of the Technical Information Center is to provide the broadest dissemination possible of information contained in DOE's Research and Development Reports to business, industry, the academic community, and federal, state and local governments.

Although a small portion of this report is not reproducible, it is being made availabble to expedite the availability of information on the research discussed herein. 


\section{LA-UR89-167}

LA-UR- $-89-167$

DE89 006530

\section{Received by bisil}

$1: .$. i) $r .1989$

Jimuilry $16-\because(1,1989$

l.os Anpelon, 1.,1l1iornl:1

\section{IDIS' I.AIMF.R}

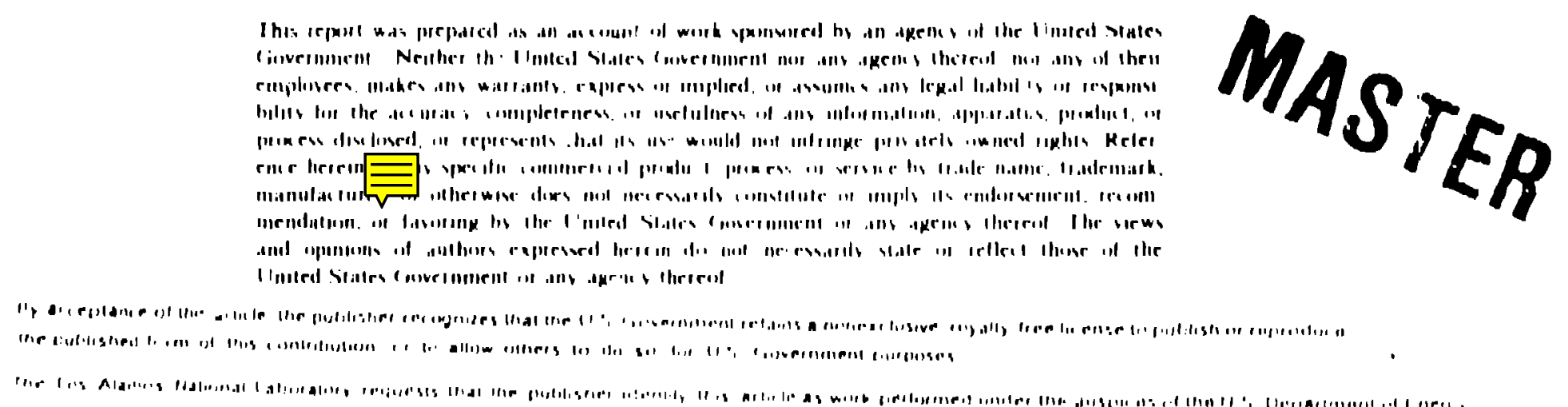

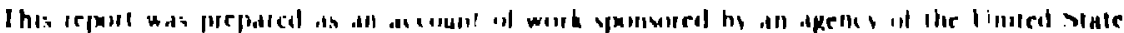

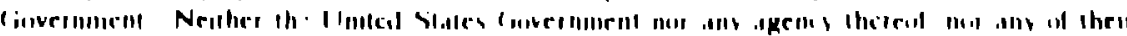

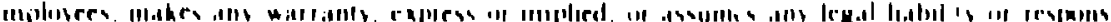

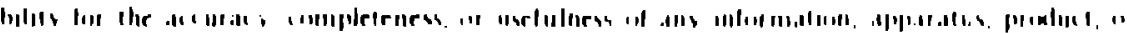
然

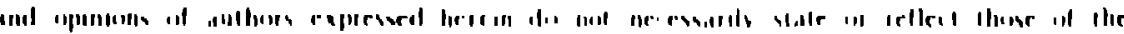

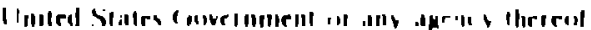




\title{
Bunch Beam Production and Mircrowave Generation in Reditrons
}

\author{
Thomas J. T. Kwan, Harold A. Davis, Robert D. Fulton, and Eugene G. Sherwood
}

\author{
Los Alamos National Laboratory \\ P. O. Box 1663 \\ Los Alamos, NM 87545
}

\begin{abstract}
We have discovered in our two-dimensonal particle-in-cell simulations that the oscillation of virtual cathodes in reditrons can produce a highly modulated electron beam. Full (100\%) current modulation of the leakage electron tsam was observed in our simulations. The modulation is at the frequency of the oscillating virtual cathode and the transverse magnetic mode generated by the reditron. We had further incorporated an inverse diode in the reditron and showed that the kinetic energy of the modulated electron beam was efficiently converted into transveise electromagnetic waves. Our simulations showed an efficiency of $26 \%$ and the time averaged microwave power was about $6 \mathrm{GW}$.
\end{abstract}

\section{LINTRODUCTION}

The reditron ${ }^{1-5}$ (reflected electron discrimination microwave generator) is a new type of virtual cathode device which uses an electron range thick anode to prevent reflected electrons from re-entering the diode reg:on. As a result, the microwave generation is purely due to oscillation of the virtual cathode in a reditron. It has been shown in our computational and experimental study that reditrons can produce microwaves with a much better speitral purity and with much higher efficiency in comparison with other virtual cathode devices. It has been demonstrated experimentally that the reritron can produce 2.3 to $3.3 \mathrm{GW}$ of microwave radiation at 7.5 to $10.0 \%$ efficiency with a very narrow spectrum centered at $2.1563 k^{6} 7$. The spectral width was predominantly limited by the number of cycles of microwaves produced in the pulse (about $f(x)$ cycles). This is ronghly a factur 
of 2.5 to 3.3 increase in efficiency and about 3 in bandwidth narrowing over conventional vircators.

In our recent simulation study, we discovered anoth.er important aspect of the redition that the transmitted electron beam current can be highly modulated by the oscillatory characteristics of the virtual cathode. Furthermore, we shewed in our computer simulations that through the use of an inverse diode that a modulated electron beam can be effectively converted into coherent transverse electromagnetic waves. The result is very enconraging that an overall efficiexzy of TEM wave production reached $26 \%$. The use of an inverted diode to extrac! power from a modulated electron beam is only one of the many methods available to us. For example, the use of a slow wave structure can lead to strong excitation of transverse magnetic modes. Incorporation of such an efficient device into the reditron design can greatly enhance the operating power level and its efficiency.

\section{IL PRODUCTION OE HIGHILYMODULATED ELECIRON BEAMS IN REDITRONS}

The reditron configuration has a number of parameters which can be varied 10 optimize output power. Among them are the anode-cathode gap, the anode slot width, and the applied magnetic field. In previous work ${ }^{6}$, the optimum anode-cathude gap was determined to be $3.7 \mathrm{~cm}$. Of course, the optimum values change with respect to electron beam voltage and current and the geometric dimension of the device. We used the optimal configuration to investigate the modulation of the leakage electron beam and the extraction of coherent electromagnetic radiation in a inverse diode coniiguration.

We used the two dimensional fully electromagnetic and relativistic particle simulation code ISIS, to study an optimized operation of the reditron. The pararaters of the case simulated were: $V_{0}$ $=1.2 \mathrm{MV}, \mathrm{l}_{\mathrm{o}}=19 \mathrm{kA}, \mathrm{R}_{\mathrm{w}}=9.0 \mathrm{~cm}, R_{\mathrm{b}}=3.0 \mathrm{~cm}, B_{\mathrm{o}}=6.1 \mathrm{kG}, \Delta_{\mathrm{ak}}=3.7 \mathrm{~cm}, \delta=0.35 \mathrm{~cm} . \mathrm{V}_{\mathrm{o}}$

is the beam voltage. $I_{0}$ is the beam current. $R_{w}$ is the waveguide radius. $k_{b}$ is the radius of ihe annular electron beam. $\Delta_{a k}$ is the anode-cathode gap. $\delta$ is the slot size of the anode. $B_{0}$ is the magnetic field of the external coil in the diode region. The magnetic field profile of field coil wits calculated according to various experimental situations by the time independent code, BIIIIL D. The' external magnetic field in a particle in cell simulation of reditrons was initialized according to th: result from the cede BIILILD. The space charge limiting current in the wavegiade section is calculated to be approximately $12 \mathrm{k} A$ according to the formula for infinitesimally thin annular electront 
beam. A TEM wave was launched from the left side into the coaxial line formed by the cathode and the anode as depicted in Fig. 1. Electron emission from the cathode was modelled according to the space charge limited field emission process. The phase space diagrams of the electron beam at an instant of time are shown in Fig. 1. The formation of a strong oscillating virtual cathode is evident. The reflexing electrons were virtually eleminated by the thick slotted anode since the chance for an electron to go through the slot several times was negligibly small. The diverging clectron beam in the waveguide was a result of the magnetic field lines of the external coil. In the simulation, the voltage had a linear rise and was then kept constant thereafter. Strong and coherent excitation of the waveguide mode $\mathrm{TM}_{01}$ was observed. The time history of the electric component of the $\mathrm{TM}_{01}$ mode and its Fourier spectrum are shown in Fig. 2. The frequency was $1.9 \mathrm{GHz}$. The bandwidth was basically limited by the pulse length of the microwave radiation in the simulation. The radiation power in the $\mathrm{TM}_{01}$ mode in the waveguide was monitored during the course of the simulation. Figure 3 snows the time evolution of the axial Poynting vector associated with the $\mathrm{TM}_{01}$ mode and its energy. The high-power and high-efficiency characteristics of the reditron were clearly demonstrated. Note that the simulation was only run out to 9.45 ns to illustrate the trend in the operation of the reditron.

We have also discovered in the computer simulation that the leakage electron beam was highly modulated due to the oscillatory characteristics of the virtual cathode. The virtual cathod: behaved as a gate controlling the transmitted electrons at the oscillating frequency. The current of the leakage electron beam was monitored in time in the simulation at thiee different axial positions and the results are shown in Fig. 4. Almost full modulation (100\%) of the electron beam was achieved immediately beyond the virtual cathode. The strong and coherent modulation of the leakage current in reditrons is significant that further extraction of radiation power from the modulated electron beam can greatiy enhance the power level and efficiency of reditrons. The frequency of modulation is that of the $\mathrm{TM}_{01}$ mode, which is the oscillating frequency of the virtual cathode. Unlike other schemes producing modulated electron beams, the leakage electron beam does not need any drift region to achieve full modulation. This discovery in the reditron stegests the incorporation of an inverse diexde or any suitably chosen slow wave structures to further increase the microwave power and production efficiency.

\section{WL. MISBUWAVE GLNERATIUN IN RLDITRUNS}


One of the more straight forward ways to extract coherent radiation from a moculated electron beam is the use of an inverse diode. The center conductor in the coaxial configuration serves as the electron beam dump and the voltage developed between the inner and outer conductors is transmitted along the coaxial line in the form of a TEM wave. Figure 5 shows a computer simulation where a center conductor was inserted in the waveguide to intercept the modulated electron beam so that the its kinetic energy would be converted to a transverse electromagnetic (TEM) wave in the coaxial transmission line. The simulation parameters are the same as the optimal case for the reditron described in the previous section. The leakage electron beam was found to be highly modulated. In Fig. 6, the time evolution of the leakage electron beam obtained by a probe at an axial position before ihe center conductor showed strong and coherent modulation. The frequency was the characteristic oscillation frequency of the virtual cathode and the strongly excited $\mathrm{TM}_{01}$ mode. The amplitude of the TEM waves as a function of time is shown in Fig. 7 indicating strong excitation of TEM waves at the frequency of $1.91 \mathrm{GHz}$ in the coaxial transmission line. This frequency corresponded to the electron beam modulation and the $\mathrm{TM}_{01}$ mode excited by the reditron. The time averaged power of the TEM wave was about $6 \mathrm{GW}$ which was $26 \%$ of the electron beam power in the diode. The axial power flow of the TEM waves in the coaxial uansmission line at three axial positions from the simulation is shown in Fig. 8.

Interacion of a modulation eiectron beam with slow wave structures leading to generation of high power microwaves is a reasonably well established subject. Usually, the slow wave structure. caluses bunching of the electron beam and electromagnetic waves are generated through an instability involving a negative energy space : harge beam mode. A highly pre-modulated electron beam would have the advantage of induring the instability at a pre-selected frequency and therefore the bandwidth of the unstable waves becomes much narrower. The reditron has already been shown to generate microwaves with narrow bandwidth and it would be natural to integrate it with the interactuon between a suitably chosen slow wave structure and the highly modulated leakage electron beam. This could lead to greally enhanced operating power level and overall efficiency.

\section{CONCLUSIONS}

We have shorve that the reditron can prexduce a highly mexdulated electron beam in additum in 
high-power microwaves. It is further demonstrated the possibility of the conversion of the modulated electron beam into electromagnetic energy, in particular, through the use of an inverse diode. By properly integrating the two mechanisms, one might realize a modified reditron which can be operated at even higher power level and efficienry.

\section{BEFERENCES}

1. T. J. T. Kwan, Phys. Rev. Lett. 571895 (1986).

2. H. A. Davis, R. R. Bartsch, T. T. T. Kwan, R. M. Stringfield, and E. G. Sherwood, Phys. Rev. Lett. 52,288 (1987).

3. T. J. T. Kwan and H. A. Davis, IEEE Transactions on Plasma Science, Vol. 16, No. 2, April 1988.

4. H. A. Davis, R. R. Bartsch, T. J. T. Kwan, E. G. Sherwood, and R. M. Stringfield, IEEE Transactions on Plasma Science, Vol. 16, No. 2, April 1988.

5. T. J. T. Kwan, H. A. Davis, R. D. Fulton, and E. G. Sherwood in Prodeedings of the Seventh International Conference on High Power Beams, Karlsruhe, Germany, July 1988.

6. T. J. T. Kwan, H. A. Davis, R. D. Fulton, and E. G. Sheriwocd, SDIO/IST 1987 Progress Report, Los Alamos National Laboratory Report L.A-UR-88-129. Dec. 1987.

7. T. J. T. Kwan, H. A. Davis, R. D. Fulton, and E. G. Sherwood, in Proceedings: of SPIE, Vol. 873, Microwave and rarticle Beam Sources and Propagation, Los Angeles, CA, 13-15 Jan. 1988. 

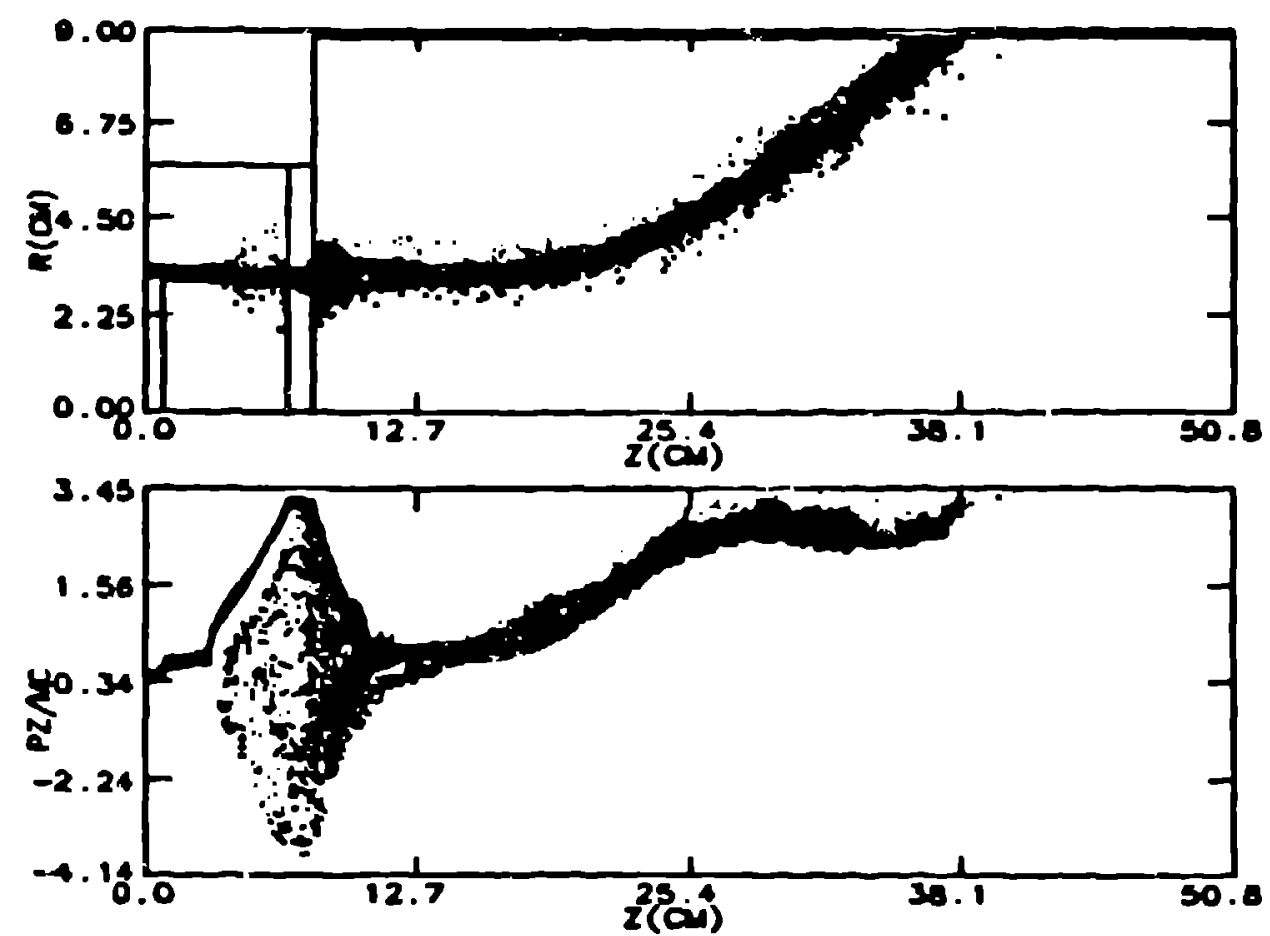

Fig. 1 Real and phase space diagrams of the optimized redtron.
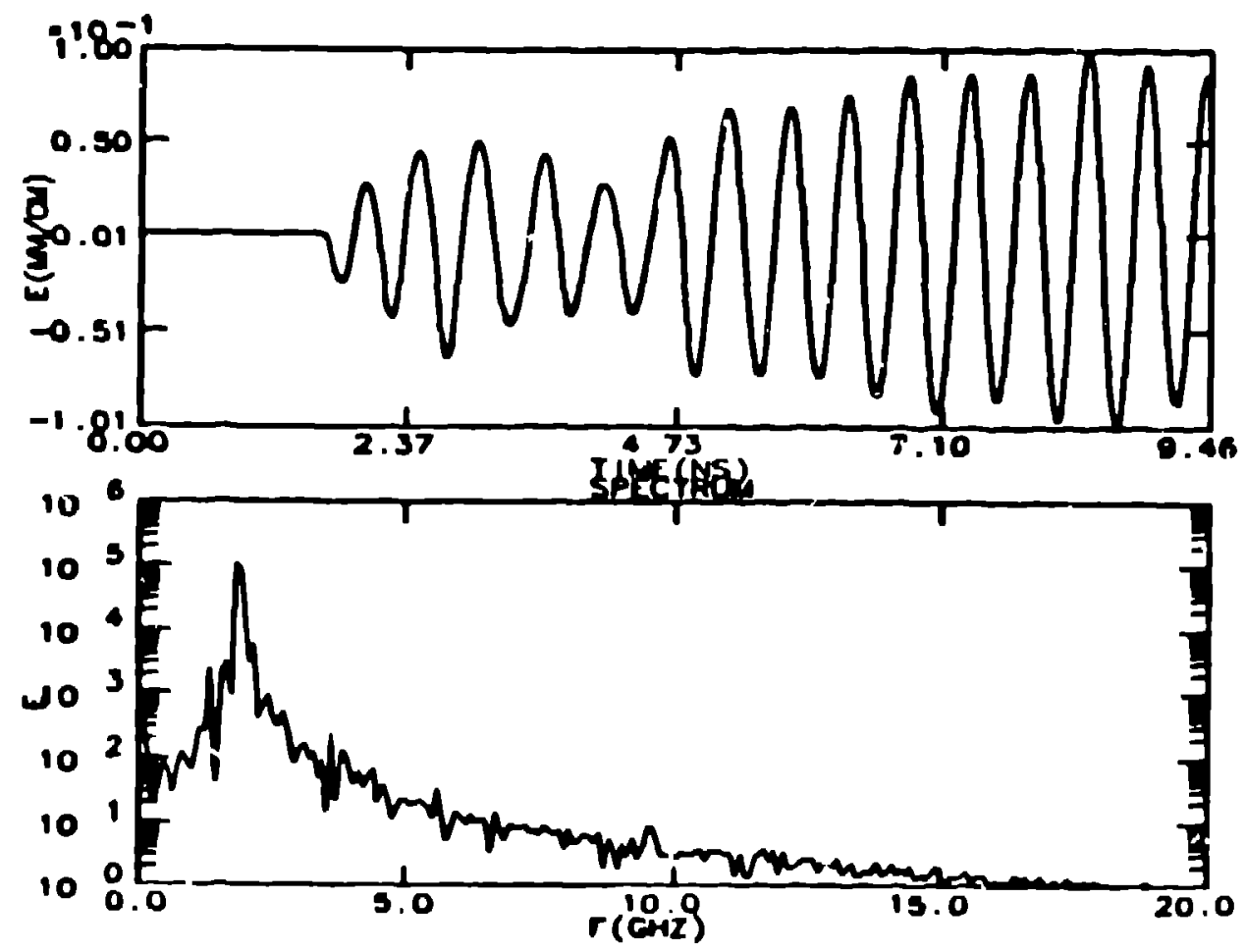

Fig. 2 Time history and Fourier spectun of $\mathrm{TM}_{01}$ from the simulation. 

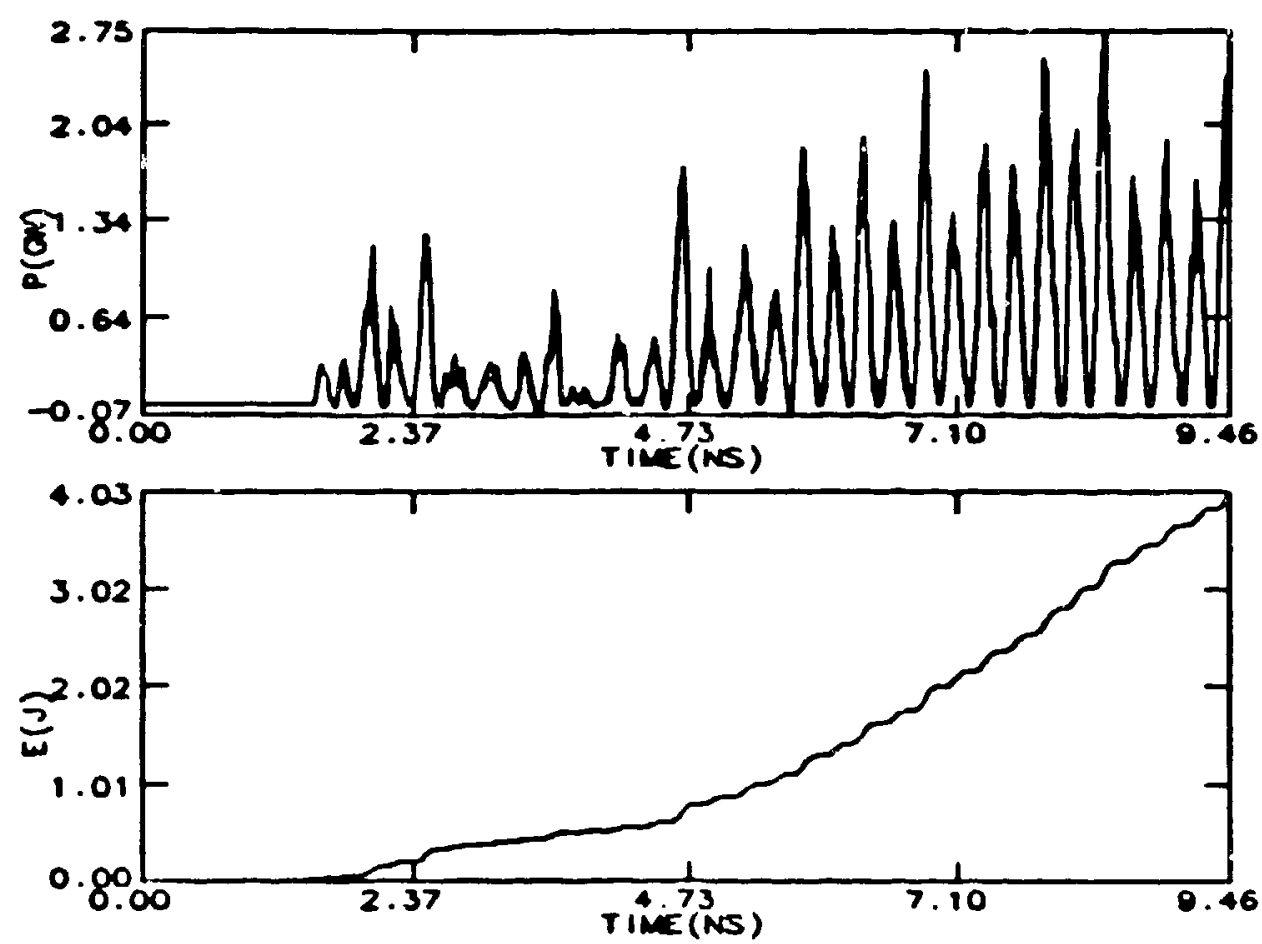

Fig. 3 Time histories of microwave power and energy produced in the optimized reditron.
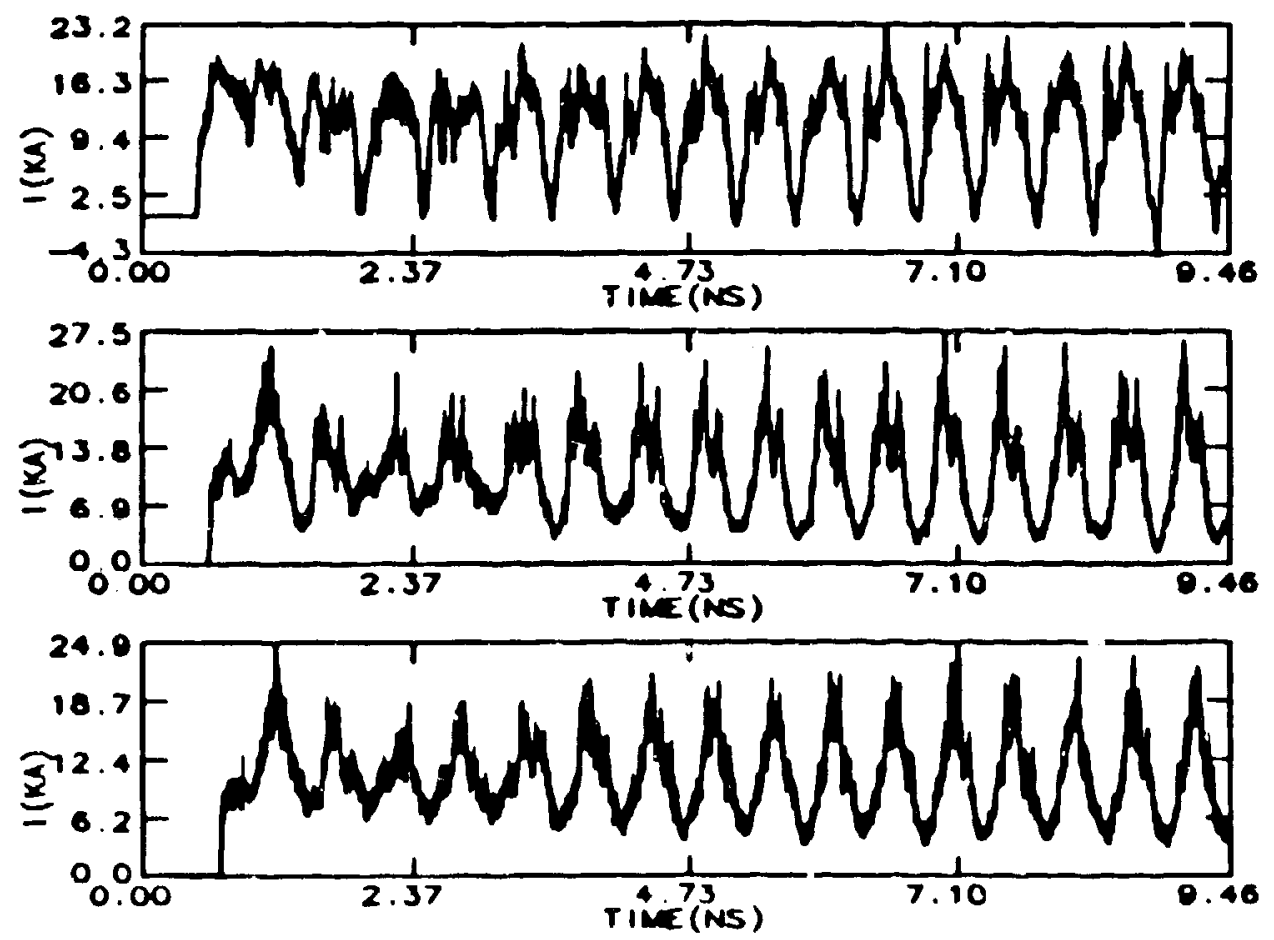

Fig. 4 Modulated electron beam produced by the reditron. 

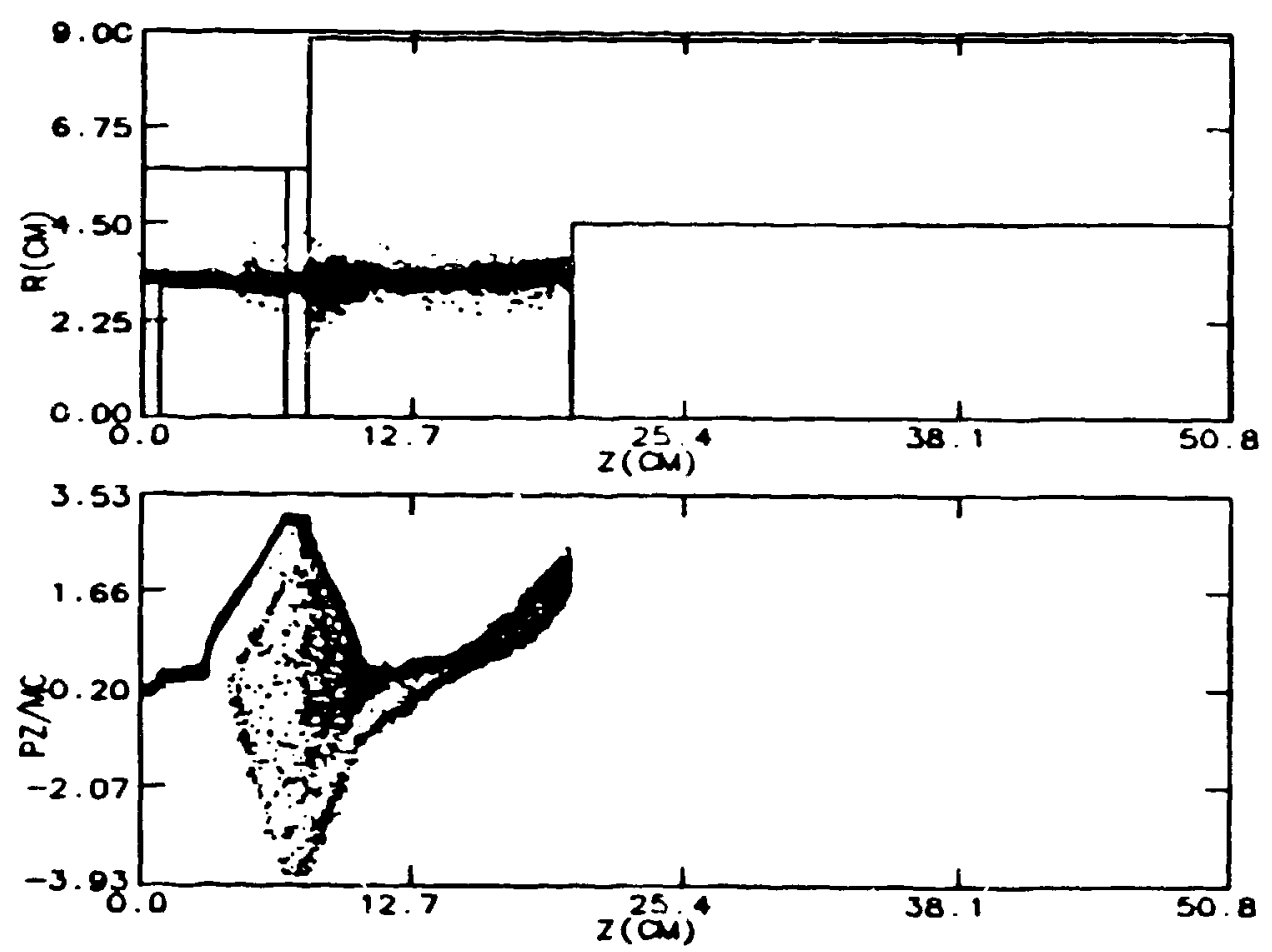

Fig.5 Incorporation of an inverse diate in the reditron.

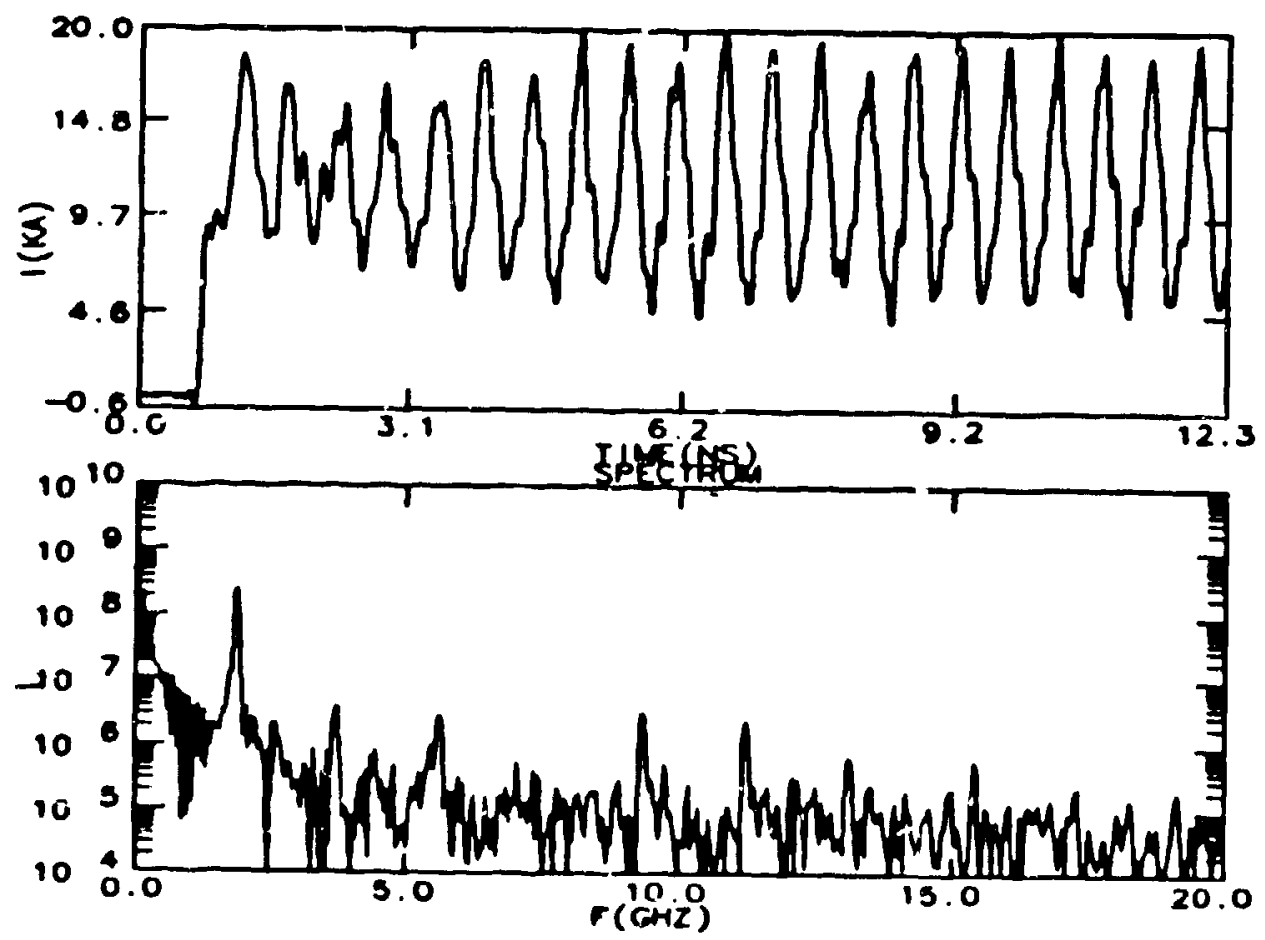

Fig. 6 Current moduiation of the transmitted beam is not affecter by the center conductor. 

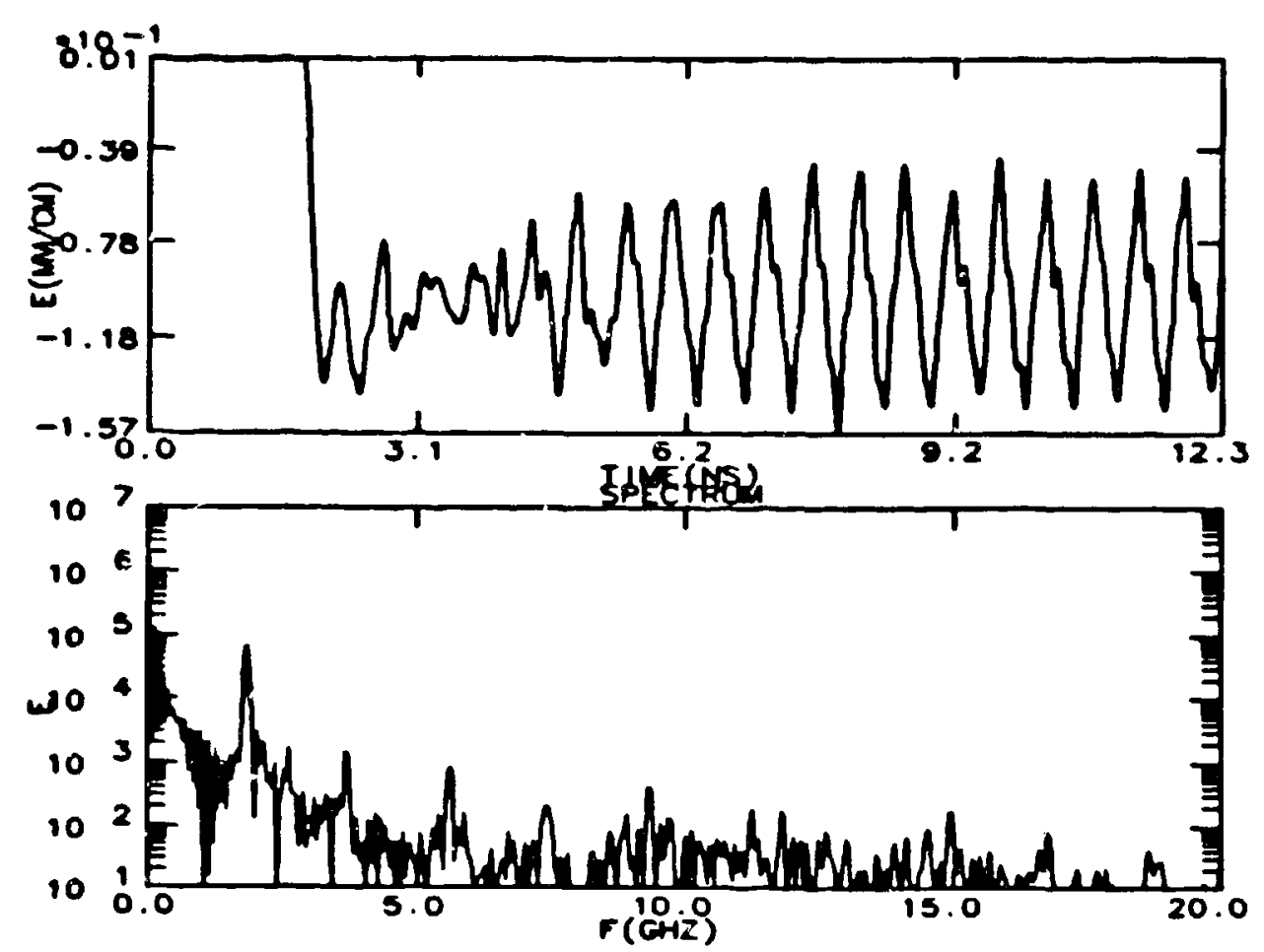

Fig. 7 Strong excitation of TEM wave in the reditron with an inverse diode.
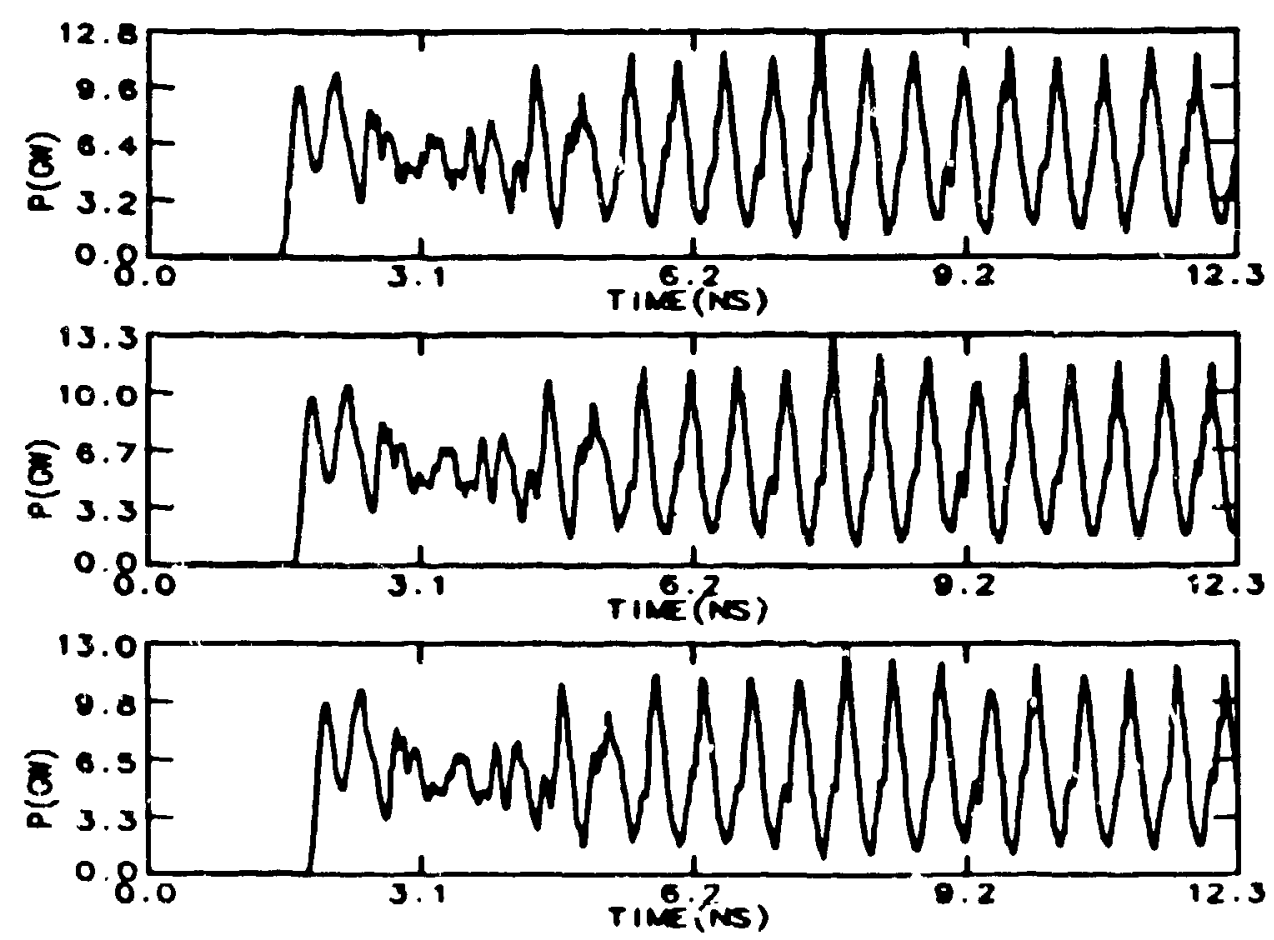

Fig. 8 Power flow of the TEM waves at three different axial positions in the coaxial transmission line. 